\title{
Trastornos mentales en hombres y mujeres expuestos a abuso sexual en la infancia
}

\author{
Cristina Haydée Arrom Suhurt ${ }^{\mathrm{I}}$, María del Pilar Fresco Arrom ${ }^{\mathrm{I}}$, Carmen Marina Arrom Suhurt ${ }^{\mathrm{II}}$, \\ Margarita Samudio ${ }^{\mathrm{III}}$, María Auxiliadora Arrom de Orrego ${ }^{\mathrm{II}}$, Andrés Claudelino Arce Ramírez ${ }^{\mathrm{I}}$, Mirta \\ Mercedes Romero Núñez ${ }^{I}$ \\ I. Cátedra de Psiquiatría-Facultad de Ciencias Médicas de la Universidad Nacional de Asunción, Paraguay \\ II. Centro para el Desarrollo de la Investigación Científica CEDIC/Díaz Gill, Paraguay \\ III. Instituto de Investigaciones en Ciencias de la Salud-Universidad Nacional de Asunción (IICS-UNA), \\ Paraguay
}

Cómo referenciar este artículo/ How to reference this article:
Arrom C, Fresco MP, Arrom CM, Samudio M, Arrom MA, Arce A, Romero MM. Trastornos mentales en hombres y mujeres expuestos a abuso sexual en la infancia. Mem. Inst. Investig. Cienc. Salud. $2015 ; 13(3): 17-23$

\section{RES U M E N}

El abuso sexual es una forma de violencia que afecta a personas de diferentes países, estratos sociales, culturas y religiones. El presente estudio se propuso analizar los antecedentes de abuso sexual infantil en adultos que consultaron en el Servicio Ambulatorio de la Cátedra de Psiquiatría y que presentaron distintos tipos de trastornos mentales. Es un estudio trasversal, descriptivo, muestreo no probabilístico. Se realizó la revisión de 254 fichas clínicas de pacientes en psicoterapia entre 2010 y 2012 y se seleccionaron 44 víctimas de abuso sexual infantil (ASI). Los diagnósticos de trastornos mentales fueron realizados en base a la clasificación DSM. El 95,4\% de los pacientes $(n=42)$ era del sexo femenino, 31,8\% $(n=14)$ tenía entre 19 y 15 años de edad, $38,6 \%$ $(n=17)$ entre 26 a 35 años y $72,7 \%(n=32)$ era de estado civil soltero. Además del ASI, el $29,5 \%$ (13) recibió violencia de pareja, de otros parientes y fuera del hogar, $25 \%$ (11) de padres, otros familiares y fuera del hogar, $25 \%$ (11) pareja, otros familiares, fuera del hogar, $4,5 \%$ (2) padres, pareja, otros familiares y fuera del hogar, 15,9\% (7) fuera del hogar. El 36,4\% (16) tenía trastornos del estado de ánimo, 18,2\% (8) de ansiedad, 15,9\% (7) de personalidad, 4,5\% (2) psicóticos, $13,6 \%$ (6) dos o más trastornos, y $6,8 \%$ (3) patología dual. Es una población de mujeres jóvenes, solteras, víctimas de abuso sexual y padecieron fundamentalmente trastornos del estado de ánimo, de ansiedad y de personalidad.

Palabras clave: abuso sexual, trastornos mentales, población adulta.

\section{Mental disorders in men and women who suffered sexual abuse or other types of violence}

\section{A B S T R A C T}

Sexual abuse affects people from different social classes, independently of their culture and religion, in all countries. This study aimed to analyze the sexual abuse suffered by adults who attended the outpatient service of the Department of Psychiatry of the Faculty of Medical Sciences. It is a cross-sectional descriptive study with non-probability sampling. It analyzed 254 clinical records of patients who attended the service for psychotherapy between 2010 and 2012 and 44 victims of child sexual abuse (CSA) were selected. The diagnoses of the mental disorders were made using the DSM classification. The $95.4 \%$ (42) was women, $31.8 \%$ (14) was between 19 and 25 years old, 38.6\% (17) between 26 and 35 and $72.7 \%$ (32) was single Apart from CSA, 29.5\% (13) received violence from partners, relatives and outside of the home, $25 \%$ (11) from their parents, relatives and outside of the home, $4.5 \%$ (2) from parents, partners, relatives and outside of the home 
and $15.9 \%$ (7) outside of the home. The $36.4 \%$ (16) had mood disorders, $18.2 \%$ (8) anxiety disorders, $15.9 \%$ (7) personality disorders, $4.5 \%$ (2) psychotic disorders, $13.6 \%$ (6) two or more disorders and $6.8 \%$ (3) dual pathology. This is a population of young single women that received sexual abuse and basically suffered mood, anxiety and personality disorders.

Keywords: sexual abuse, mental disorders, adult population.

\section{INTRODUCCIÓN}

El abuso sexual es una forma de violencia que adquirió mayor visibilidad en las últimas décadas (1). Para UNICEF, el abuso sexual infantil se define como: contactos e interacciones entre un niño y un adulto, cuando el adulto agresor usa al niño para estimularse sexualmente a sí mismo o a otra persona (2). La OMS describe la violencia sexual como cualquier acto sexual, tentativa de consumar un acto sexual u otro acto dirigido contra la sexualidad de una persona mediante coacción por otra persona, independientemente de su relación con la víctima, en cualquier ámbito. Comprende la violación, que se define como la penetración, mediante coerción física o de otra índole, de la vagina o el ano con el pene, otra parte del cuerpo o un objeto (3).

Aún en la actualidad prevalecen algunas creencias falsas respecto al abuso sexual, lo cual contribuye a ocultar el problema (1). Afecta en todos los países a personas de diferentes estratos sociales, independientemente de su cultura y religión. En todo el mundo, UNICEF estima que el $20 \%$ de las mujeres y entre el 5 y el $10 \%$ de los hombres sufrieron abuso sexual en su infancia (4).

Algunos aspectos que han motivado el interés de la psiquiatría actual por la temática del abuso sexual guardan relación con la psicopatología que presentan pacientes adultos con antecedentes traumáticos infantiles. Historias de trauma infantil y abuso sexual se obtienen más frecuentemente de lo esperado en pacientes con psicopatología, particularmente con trastornos afectivos, trastornos ansiosos, trastornos por alimentación, trastornos por somatización y trastornos de personalidad (5). También se observan trastornos por estrés post traumático, insomnio e intentos de suicidio (3). A esto se suma el hallazgo frecuente de estos antecedentes en pacientes que consultan en servicios de medicina por cuadros como colon irritable, cefalea y síntomas ginecológicos $(6,7)$. Y finalmente no se deben dejar de lado los riesgos de lesiones, infecciones de trasmisión sexual, aumento del consumo de sustancias, embarazos no deseados, abortos y otras repercusiones que se pueden presentar (3).

Algunas estimaciones muy precisas de prevalencia de violencia de pareja y violencia sexual son las de la OMS que basada en un estudio sobre la salud de la mujer en 10 países reveló que el $35 \%$ de las mujeres había sufrido violencia física o sexual por parte de su pareja o violencia sexual por terceros (3). Un estudio del Centro Paraguayo de Estudios de la Población (8) a nivel nacional estimó que el 6,5\% de las mujeres de 15 a 44 años fue víctima de abuso sexual (8). Una investigación realizada en Alto Paraná con una población de estudiantes de colegios públicos y privados encontró una prevalencia de $8,6 \%$ de exposición al abuso sexual con una muestra representativa de las cuatro ciudades más pobladas del Departamento (9). Otro estudio realizado en la Cátedra de Psiquiatría de la Universidad Nacional de Asunción, con una población en edad pediátrica (de 3 a 18 años) $9,2 \%$ de exposición a abuso sexual y otras formas de violencia (10). Una investigación con mujeres puérperas de un Hospital Público en Asunción evidenció que 8,6\% había sido víctima de abuso sexual y este abuso se asoció significativamente a episodios depresivos padecidos por las víctimas (11). Otro hallazgo de $8 \%$ de abuso sexual en la relación de pareja íntima y $6 \%$ de intento de abuso sexual se encontró en una población de mujeres que consultó en el Hospital Distrital de Lambaré en el 2010 (12). Por último, un estudio encontró que 18,9\% de pacientes adultos de la Cátedra de Psiquiatría de la UNA señaló haber sido víctima de abuso sexual sola o combinada con otras formas de violencia entre el 2010 y 2012 (13).

En Paraguay, vivir sin violencia es un derecho consagrado y garantizado en la constitución nacional (14). Visibilizar y contextualizar las consecuencias de la violencia, generar estadísticas que permitan cuantificar los casos y poder aplicar los procesos legales son tareas urgentes que se deben realizar. Sin embargo, por el momento los esfuerzos son 
insuficientes (15), por lo que se refuerza la necesidad de aumentar las investigaciones en el área. El presente estudio se enfoca en analizar el abuso sexual sufrido por hombres y mujeres que acudieron al servicio ambulatorio de la Cátedra de Psiquiatría de la Facultad de Ciencias Médicas y fueron derivados a psicoterapia durante el período 2010-2012.

\section{PACIENTES Y MÉTODOS}

Es un estudio de diseño descriptivo, de corte trasversal, Como el objetivo del estudio pretendía enfocarse en una población adulta víctimas de abuso sexual infantil que había desarrollado distintos tipos de trastornos mentales en la edad adulta, se realizó la selección de la población, analizando 254 fichas clínicas de pacientes remitidos a psicoterapia entre enero del 2010 a marzo del 2013 al servicio ambulatorio. Para la identificación del abuso sexual, fue utilizada la definición de UNICEF (16), como "relaciones sexuales forzadas, impuestas mediante amenazas e intimidaciones o con la fuerza física, la coerción a prácticas sexuales indeseadas, o la constricción a tener relaciones sexuales con terceros". Se buscó además en la población víctima de abuso, padecimiento de violencia física, entendida como "bofetadas, golpes, torsión de brazos, puñaladas, estrangulación, quemaduras, sofocación, patadas, amenazas con armas u otros objetos, y en casos extremos el asesinato (16) y violencia psicológica como "intimidar, atormentar a la víctima y asume diferentes formas: amenazas de abandono o abuso, reclusión en el hogar, vigilancia estricta, amenazas de destitución del cuidado de los hijos, destrucción de objetos, aislamiento, agresiones verbales y humillaciones constantes". (16) Para diagnosticar los trastornos mentales en Eje I y II se utilizaron los criterios propuestos por el Manual Diagnóstico y Estadístico (DSM IV TR) de la Asociación Americana de Psiquiatría. Otros datos analizados fueron las características socio-demográficas (edad, sexo, estado civil).

La información contenida en las fichas fue cargada en una base de datos en Excel y para el análisis de los datos, se utilizó el paquete estadístico SPSS versión 15.0 para Windows, con estadística descriptiva para expresar los resultados.

\section{RESULTADOS}

De los 44 pacientes estudiados, el 95,5\% ( $n=42)$ eran mujeres, el 34\% (15) tenía entre 19 y 25 años de edad. El 72,7\% (32) era de estado civil soltero (Tabla 1 ).

Tabla 1. Distribución de la población por rango de edad, sexo y estado civil. $n=44$

\begin{tabular}{lll}
\hline Características & $\mathbf{n}$ & $\mathbf{\%}$ \\
\hline Sexo & 2 & 4,5 \\
$\quad$ Masculino & 42 & 95,5 \\
$\quad$ Femenino & & \\
Rangos de Edad (años) & 15 & 34 \\
19 a 25 & 17 & 39 \\
26 a 35 & 8 & 18 \\
36 a 45 & 3 & 7 \\
46 a 55 & 1 & 2 \\
> 55 & 32 & 72,7 \\
Estado Civil & 5 & 11,4 \\
$\quad$ Soltero/a & 1 & 2,3 \\
En pareja/casado/a & 6 & 13,6 \\
$\quad$ Divorciado/Separado/a & & \\
Sin datos &
\end{tabular}

El $61,4 \%(n=27)$ de la población fue víctima de abuso sexual combinado con violencia física y psicológica; y de este total, $56,8 \%$ (25) fueron mujeres y $4,5 \%$ (2) varones (Tabla 2). 
Tabla 2. Tipo de violencia por sexo. $n=44$

\begin{tabular}{llll}
\hline Tipo de Violencia & Mujer & Varón & Total \\
\hline Abuso sexual, violencia física y psicológica & $25(56,8)$ & $2(4,5)$ & $27(61,4)$ \\
Abuso sexual & $9(20,5)$ & 0 & $9(20,5)$ \\
Abuso sexual y violencia psicológica & $8(18,2)$ & 0 & $8(18,1)$ \\
\hline
\end{tabular}

El $86,4 \%(n=38)$ recibió violencia del entorno familiar que incluye padre, padrastro, hermanos, tíos, primos, cuñado, hermanastro, niñera, novio, amigos de la familia. (Tabla 3).

Tabla 3. Características del agresor. $n=44$

\begin{tabular}{lll}
\hline Agresor & $\mathbf{n}$ & $\mathbf{\%}$ \\
\hline Entorno familiar (padre, padrastro, hermanos, tíos, primos, & 38 & 86,4 \\
cuñado, hermanastro, niñera, novio, amigos de la familia) & 4 & 9,1 \\
Extraños & 2 & 4,5 \\
Entorno laboral (quehaceres domésticos) & 2 & \\
\hline
\end{tabular}

El 36,4\% (16) tenía trastornos del estado de ánimo, 18,2\% (8) de ansiedad, 15,9\% (7) de personalidad, $13,6 \%$ (6) dos o más trastornos, 6,8\% (3) patología dual 4,5\% (2) con trastornos psicóticos y $4,5 \%$ (2) otros (Tabla 4).

Tabla 4. Distribución de la población por tipo de Trastornos. $n=44$

\begin{tabular}{lll}
\hline Tipo de Trastornos & $\mathbf{n}$ & $\mathbf{\%}$ \\
\hline Trastornos del estado de ánimo & 16 & 36,4 \\
Trastornos de ansiedad & 8 & 18,2 \\
Trastornos de personalidad & 7 & 15,9 \\
Dos o más trastornos & 6 & 13,6 \\
Patología dual & 3 & 6,8 \\
Trastornos psicóticos & 2 & 4,5 \\
Otros trastornos & 2 & 4,5 \\
\hline
\end{tabular}

La Tabla 5 muestra el tipo de violencia sufrida por los pacientes que presentaron trastornos del estado de ánimo y de ansiedad.

Tabla 5. Abuso sexual en pacientes con trastornos del estado de ánimo y de ansiedad.

\begin{tabular}{lll}
\hline Violencia sufrida & $\begin{array}{l}\text { Trastornos del } \\
\text { estado de animo }\end{array}$ & $\begin{array}{l}\text { Trastornos de } \\
\text { ansiedad }\end{array}$ \\
\hline Abuso sexual, violencia física y psicológica & $10(62,5)$ & $4(50)$ \\
Abuso sexual y violencia psicológica & $2(12,5)$ & $4(50)$ \\
Abuso sexual & $4(25)$ & 0 \\
\hline
\end{tabular}




\section{DISCUSIÓN}

El abuso sexual al cual fue expuesta la población en estudio, recayó principalmente en las mujeres, lo que coincide con datos de investigaciones precedentes sobre la asociación significativa entre género femenino y población víctima de violencia, principalmente abuso sexual $(3,17)$, información que se ha expuesto también en estudios nacionales $(10,13,18)$. En Colombia el hallazgo fue en una investigación sobre más de 18.000 dictámenes sexológicos donde el $84 \%$ de los afectados por abuso sexual fueron mujeres (19).

En el presente estudio se constata que mujeres y hombres adultas/os, mayoritariamente jóvenes con antecedentes de abuso sexual en la infancia, presentan diversas afecciones mentales en la actualidad. La violencia sexual, está demostrado, produce un deterioro profundo del bienestar físico, sexual, reproductivo, psíquico, mental y social de las personas y las familias. Y entre las consecuencias a largo plazo sobre la salud se citan el trastorno de estrés postraumático y la depresión $(11,13,18)$. Estudios realizados en el país muestran que los padecimientos mentales se vinculan a la presencia de diferentes formas de violencia recibida por las personas en distintos momentos de sus vidas (10-13). A más del abuso sexual, en estudios de referencia en Paraguay se encontró en poblaciones expuestas a abuso sexual, que también habían sido víctimas de otras formas de violencia en el ámbito familiar de parte de sus parejas y familiares. (10-13).

El parentesco entre víctima y agresor queda profundamente evidenciado en esta población de adultos que consultó al Servicio de Psiquiatría y es un hallazgo evidenciado en estudios que dan cuenta de que en la gran mayoría de los casos, los agresores eran conocidos de la víctima y el lugar de los hechos la vivienda de la persona agredida $(12,19,20)$. Un estudio nacional reportó que el $8,6 \%$ de una población de estudiantes secundarios fue víctima de abuso en el ámbito familiar $(9,21)$ y otro en México reportó relación entre abuso sexual ocurrido en el ámbito familiar por personas del entorno (17).

Los trastornos más encontrados en la población afectada fueron trastornos del estado de ánimo, de ansiedad (entre los que se incluyó el trastorno por estrés post traumático) y los trastornos de personalidad, lo que concuerda con literatura de referencia que establecen a la depresión, el trastorno por estrés post traumático, pánico y abuso de sustancias como los trastornos más frecuentemente encontrados en población expuesta al abuso sexual. $(11,13,21,22)$. En estudio de Ceballo et al. se resaltó la exposición a violencia con mayores reportes de depresión y síntomas de trastorno por estrés postraumático $(12,21,23)$.

La presencia de trastornos mentales en esta franja etaria, mayoría entre 19 y 35 años, que podría estar afectada por las secuelas del abuso sexual, se instala en un período de la vida caracterizado por la búsqueda de desarrollo personal, que se ve impactado negativamente en su proceso. Otros estudios nacionales evidenciaron presencia de depresión en mujeres que fueron víctimas de violencia $(12,13,21)$.

Llama la atención que dos tercios de la población estudiada se encuentre en un estatus civil de soltería. Esto diverge de lo propuesto por otros estudios que consideran el estar casado o vivir en pareja como un factor de riesgo para ser víctima de abuso o alguna otra forma de violencia $(24,25)$. Podría pensarse que el abuso desencadenaría la dificultad para establecerse en una relación de pareja. Y esta situación se vería agravada cuando hay concomitancia con otras formas de violencia como la que se observa en más de la mitad de la población estudiada y en los estudios citados en bibliografía comparada.

La violencia sexual y la violencia infligida por la pareja pueden afectar a todas las personas aunque existe una fuerte tendencia de género y hasta hoy, los estudios muestran que la mayoría de las víctimas son mujeres y la mayoría de los agresores son hombres. Este daño causado por la violencia sexual puede durar toda la vida y afectar a varias generaciones con graves consecuencias para la salud, la educación, el empleo, el bienestar económico de la persona, la familia, la comunidad y la sociedad según reportan numerosas organizaciones e instituciones $(18,26)$. En Paraguay, la violencia contra la Mujer (VcM) representa más de 20 millones de días de trabajo perdidos y más de 72.000 colaboradores/as sin producción según el estudio recientemente publicado por la Cooperación Alemana, implementado por la GIZ a través del Programa Regional Combatir la Violencia contra las Mujeres en Latinoamérica (ComVoMujer) (27) en el marco de mejorar la cooperación entre actores estatales y no estatales para combatir la violencia de género y en alianza con el sector empresarial entre otros. (27). El Informe sombra a del Comité para la Eliminación de las Discriminación contra la Mujer (CEDAW) de parte de la 
sociedad civil en Paraguay sobre el cumplimiento del Estado de la Convención sobre la Eliminación de Todas las Formas de Discriminación contra la Mujer de las Naciones Unidas en el 2011 reportaba que persisten en el país normas discriminatorias, principalmente un sesgo en la administración de justicia que impide el acceso de las mujeres tanto para acceder como en la atención y los resultados de ésta (28).

Cabe recordar finalmente en este contexto la necesidad de que Paraguay cumpla con el "Programa de 14 Puntos para la Prevención, sanción y erradicación de la violencia contra las mujeres", presentado al país por Amnistía Internacional (26).

\section{REFERENCIAS BIBLIOGRÁFICAS}

1. Casado Flores J, Díaz Huertas JA, Martínez González C. Niños maltratados. Abuso sexual: un problema desconocido. Ediciones Díaz de Santos. Madrid, España. 1997.

2. UNICEF. Conceptos vinculados a la explotación sexual infantil. /Internet/. Buenos Aires-Argentina: Fondo de las Naciones Unidas para la Infancia; /citado 4 de Mayo 2013/ Disponible en: http://www.unicef.org/argentina/spanish/de finiciones.pdf

3. Organización Mundial de la Salud. Violencia contra la mujer. Violencia de pareja y violencia sexual contra la mujer. /Internet/. Ginebra-Suiza: OMS; /citado 15 de mayo de 2013/. Disponible en: http://www.who.int/mediacentre/factsheets /fs239/es/

4. UNICEF. Secretario General de Naciones Unidas. Derechos del niño. /Internet/. Santiago de Chile: Agencias de Naciones Unidas, Fondo de las Naciones Unidas para la Infancia (UNICEF); 2006. /citado 6 de junio de 2014/. Disponible en: http://www.unicef.org/violencestudy/report s/SG_violencestudy_sp.pdf

5. Florenzano R, Fullerton C, Acuña J, Escalona R. Somatización: aspectos teóricos, epidemiológicos y clínicos. Rev Chil NeuroPsiquiat. 2002;40(1):47-55.

6. Leserman J. Sexual and physical history in gastroenterology, how the types of abuse influence the health. Psych Med. 1996;58:4-15.

7. Vitriol GV. Relación entre psicopatología adulta y antecedentes de trauma infantil. Rev Chil Neuro-Psiquiat. 2005;43(2):88-96.

8. Centro Paraguayo de Estudios de Población.Encuesta Nacional de Demografía y Salud Sexual y Reproductiva.; /Internet/. Asunción Paraguay: CEPEP; /citado 20 mayo 2014/. Disponible en: http://www.cepep.org.py/archivos/ENDSSR $\% 202008$ 1.pdf

9. Ruoti $M$, Arrom C. Diferentes formas de violencia padecidas por adolescentes durante su crianza. Revista Paraguaya de Psiquiatría. 2015;3(1):50-6.

10. Arrom C, Fresco MP, Arrom CM, Arce A, Arrom MA, Romero $M$, et al. Violencia intrafamiliar. Revista Paraguaya de Psiquiatría. 2015;3(1):38-41.
11. Arrom C, Samudio M, Arrom CM, Lampert N, Arrom MA, Orué E. Violencia intrafamiliar, de pareja íntima y abuso sexual en puerperio inmediato. Su relación con depresión. Revista Paraguaya de Psiquiatría. 2013; 1(2):17-23.

12. Galeano $A$, Orrego $B$, Pérez $M$, Ruiz $V$, Arrom $M A$, Ruoti $M$, et al. Violencia intrafamiliar sufrida por mujeres en un Hospital Público. Revista Paraguaya de Psiquiatría. 2015;3(1):42-9.

13. Fresco MP, Arrom C, Samudio M, Arrom CM, Capurro M, Arrom MA, et al. Psicopatología en adultos con antecedentes de violencia intrafamiliar. Revista Paraguaya de Psiquiatría. 2015;3(1):8-12.

14. Chamorro J, Otero H. Derechos de la Niñez y la adolescencia. Pendientes con más de veinte años para quienes todavía no tienen dieciocho. Coordinadora de Derechos Humanos del Paraguay (Codehupy). Derechos Humanos en Paraguay/ Yvypóra Derécho Paraguáipe. Codehupy. Asunción: Mercurio S.A. 2009. pp 379-92.

15. González Vera M. Diez años de la ley contra la violencia doméstica. Avances y desafíos. En: Coordinadora de Derechos Humanos del Paraguay (Codehupy). Derechos Humanos en Paraguay/ Yvypóra Derécho Paraguáipe. Codehupy. Asunción: Mercurio S.A. 2010. pp. 353-66.

16. UNICEF. La violencia doméstica contra mujeres y niñas. /Internet/. Florencia-Italia: Innocenti Digest-Unicef; /citado 2 abril 2015/. Disponible en: http://www.unicefirc.org/publications/pdf/digest6s.pdf

17. Pineda-Lucatero A. Prevalence of childhood sexual abuse among Mexican adolescents. Child: Care, Health \& Development /serial on the Internet/. /citado August 20, 2015/. 35(2):184-9. Available from: https://www.ebscohost.com/academic/psych ology-behavioral-sciences-collection.

18. Organización Mundial de la Salud-Escuela de Higiene y Medicina Tropical de Londres. Plan de acción mundial para fortalecer la función del sistema de salud en la lucha contra la violencia interpersonal, en particular la ejercida sobre las mujeres y las niñas y sobre los niños en general. /Internet/. Washington D.C.: OPS-OMS; /citado 10 Mayo 2014/. Disponible en: http://apps.who.int/iris/bitstream/10665/448 10/1/9789275316351_spa.pdf 
19. Dallos Arenales Marta Isabel, Pinzón-Amado Alexánder, Barrera González Carlos A, Mujica Rojas Johanna A, Meneses Silva Yenis R. Impacto de la violencia sexual en la salud mental de las víctimas en Bucaramanga, Colombia. rev.colomb.psiquiatr. /serial on the Internet/. 2008 Mar /cited 2015 Dec 01/;37(1):56-65. Disponible en: www.redalyc.org/pdf/806/80637105.pdf.

20. Organización Mundial de la Salud-Oficina Regional para las Américas-Organización Panamericana de la Salud. Violencia infligida por la pareja. /Internet/. Washington D.C., EEUU: OPS-OMS; /citado 2 abril 2014/. Disponible en: http://apps.who.int/iris/bitstream/10665/988 16/1/WHO_RHR_12.36_spa.pdf?ua =1

21. Arrom C, Ruoti $M$, Samudio $M$, Orué $E$ Condiciones socioeconómicas de adolescentes embarazadas con síntomas de depresión, consumo de sustancias y víctimas de violencia intrafamiliar. Revista Paraguaya de Psiquiatría. 2015;3(1):13-9.

22. Valenzuela $\mathrm{S}$. Violencia intrafamiliar como factor de riesgo en la mujer embarazada y su producto. Tesis de Grado. Colima-México: Universidad de Colima, Facultad de Medicina. 2004.

23. Ceballo R. Domestic violence and women's mental health in Chile. Psychology of Women Quarterly /serial on the Internet/. (2004, Dec), /citado 19 de agosto, 2015/; 2015; 28(4):298-308. Disponible en https://www.ebscohost.com/academic/psych ology-behavioral-sciences-collection.
24. Cantu M, Coppola M, Lindner AJ. Evaluation and management of the sexually assaulted woman. Emerg Med Clin North Am. 2003;1(3):737-50.

25. Cuesta Roldán J, Monreal Gimeno MC. Hijos e hijas víctimas de la violencia de género:importancia de una intervención directa.2012. /citado 6; de junio de 2014/. Disponible en: http://www.congresoestudioviolencia.com/20 12/articulo24.php

26. Torales J. La violencia contra las mujeres es un problema de Estado: Programa de 14 puntos para la prevención, sanción y erradicación de la violencia contra las mujeres. Revista Paraguaya de Psiquiatría. 2015;3(1):70-3.

27. Brendel C, Heikel MA. Los costos empresariales de la violencia contra las mujeres en Paraguay. /Internet/. Cooperación Alemana-GIZ. /citado 16 de setiembre de 2015/. Disponible en PDF: http://info.comvomujer.org.pe/catalogocomv o/productoscatalogos_3_2015/ComVoMujer ResumendelestudiodecostosPY_2015.pdfCED AW.

28. CODEHUPY-CLADEM-CMP-CDE-Equipo Feminista de Comunicación. Informe sombra a CEDAW Paraguay 2011./Internet/. /citado 16 de setiembre de 2015/. Disponible en PDF:

http://www2.ohchr.org/english/bodies/cedaw /docs/ngos/Joint_NGO_submission_Paraguay _CEDAW50_sp.pdf 